\title{
Backward Surface Wave Propagation and Radiation along a One-Dimensional Folded Cylindrical Helix Array
}

\author{
Bin Xu and Yang Li \\ Department of Electrical and Computer Engineering, Baylor University, Waco, TX 76798, USA \\ Correspondence should be addressed to Bin Xu; bin_xu@baylor.edu
}

Received 28 April 2015; Accepted 30 July 2015

Academic Editor: Diego Masotti

Copyright ( 92015 B. Xu and Y. Li. This is an open access article distributed under the Creative Commons Attribution License, which permits unrestricted use, distribution, and reproduction in any medium, provided the original work is properly cited.

\begin{abstract}
Wave propagation along a closely spaced folded cylindrical helix $(\mathrm{FCH})$ array is investigated for the purpose of designing compact array for energy transport and antenna radiation. It is found that the height of this surface wave guiding structure can be decreased from $0.24 \lambda_{0}$ to $0.06 \lambda_{0}$ by replacing the monopole element with the $\mathrm{FCH}$. Both the propagation constant and the mode distribution of the dominant wave mechanism are extracted by ESPRIT algorithm, which indicates that a backward propagating surface wave is supported by the array structure. A compact backfire FCH antenna array is designed and measured based on the identified dominant wave mechanism.
\end{abstract}

\section{Introduction}

Wave propagation and radiation along a one-dimensional metal wire array has long been of interest since the invention of the Yagi-Uda antenna [1]. With carefully selected monopole wire height ( 0.2 to $0.25 \lambda_{0}$, with $\lambda_{0}$ being the free space wavelength) and spacing ( 0.2 to $\left.0.35 \lambda_{0}\right)$, a surface wave with an optimum phase velocity can be supported by the structure and can be radiated in the end-fire direction [2]. More recently, there is an emerging interest in minimizing the size of the array for two important applications: electromagnetic (EM) energy transport and compact antenna array design. It was found in [3] that the transmission loss of EM energy along a $1 \mathrm{D}$ metal rod array is smaller than $1.5 \mathrm{~dB} / \lambda_{0}$, which can be attributed to the strong coupling between closely spaced rods $\left(0.054 \lambda_{0}\right)$. For the second application, a closely spaced, multiple-element parasitic antenna array was designed and implemented, with its interelement spacing being as small as $0.02 \lambda_{0}[4]$.

In spite of the above success in reducing the spacing between wires, there still remains a challenge to minimize the height of each array element. It was found that the height of metal wire should be close to a quarter-wavelength to ensure the dominant surface wave is strongly excited. One idea to minimize the element size is to substitute the metal wire with electrically small elements such as a folded spherical helix (FSH) [5] or a folded cylindrical helix (FCH) [6], the size of which can be as small as $0.06 \lambda_{0}$. The effects of mutual coupling between two closely spaced FSHs or FCHs have been studied recently for wireless power transfer applications $[7,8]$. However, wave propagation along a 1D closely spaced array with electrically small element is not well understood and still needs to be investigated. Previously we have simulated surface wave propagation along a $1 \mathrm{D} \mathrm{FCH}$ array and compared the results with that of a $1 \mathrm{D}$ metal cut wire array [9]. In this paper, we extend our study to simulate and measure surface wave propagation and radiation along a $1 \mathrm{D}$ closely spaced FCH array.

The paper is organized as follows: in Section 2, a 21element FCH array is first designed and fabricated. Broadband transmission data and near electric field distributions are simulated and measured along the array. In Section 3, the dominant propagation mechanism and its associated propagation characteristics, such as propagation constant and mode distribution, are extracted using a super resolution estimation algorithm. A parametric study is performed to figure out correlation between the propagation constant and array geometrical parameters. In Section 4 , a compact backfire parasitic antenna array is designed based on the identified dominant wave mechanism. Both simulation and measurement results are presented. Section 5 concludes the paper. 


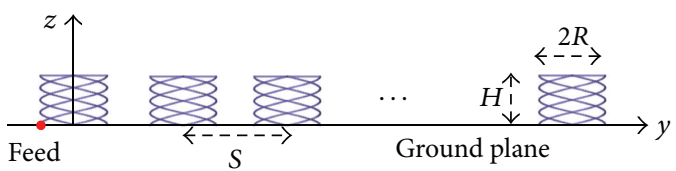

(a)

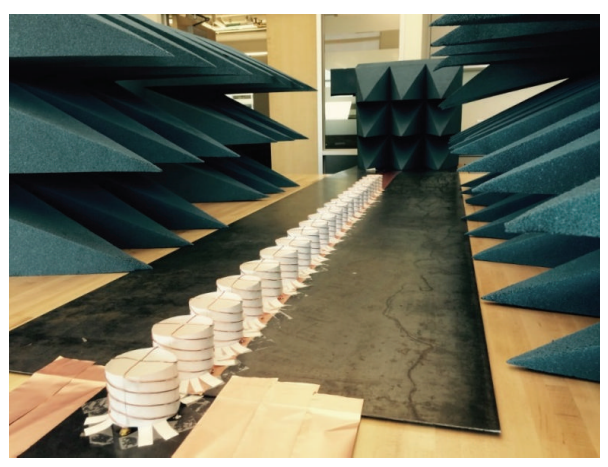

(b)

FIGURE 1: 1D folded cylindrical helix array setup: (a) simulation and (b) measurement.

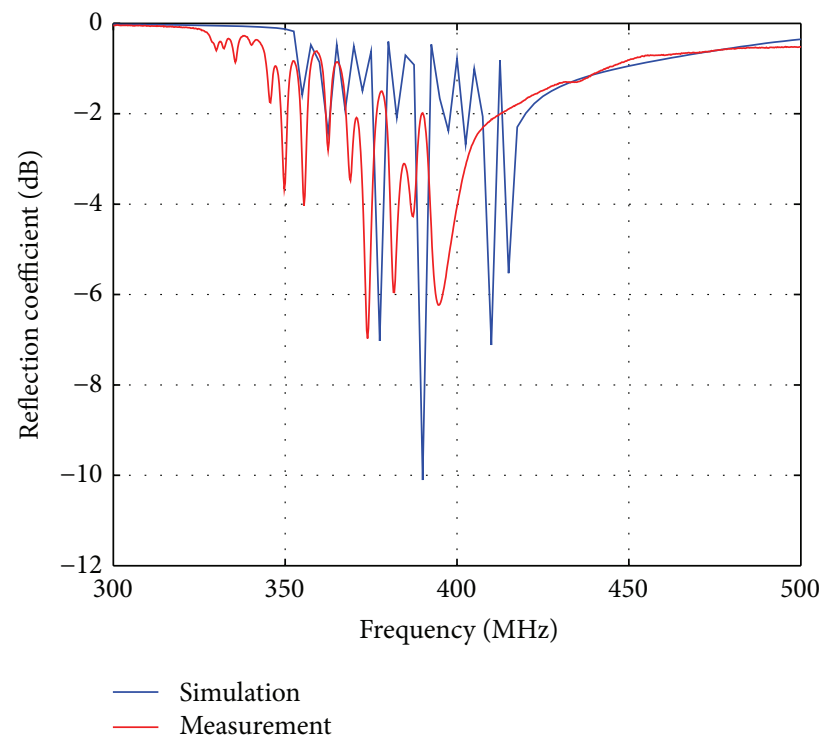

FIGURE 2: Simulated and measured reflection coefficients.

\section{Wave Propagation along a 1D FCH Array}

First, a single 4-arm FCH element centered at $400 \mathrm{MHz}$ is designed by using formulas provided in [6]. The helix radius $R$, height $H$, and number of turns are $0.0317 \mathrm{~m}$, $0.047 \mathrm{~m}$, and 1 , respectively. The height of the folded helix corresponds to $0.063 \lambda_{0}$, which is much shorter than that of a quarter-wavelength monopole antenna. Next, 21 identical elements are placed along a straight line in the $y$-axis to form the FCH array. The interelement spacing $S$ is equal to $0.08 \mathrm{~m}$, corresponding to $0.11 \lambda_{0}$ at $400 \mathrm{MHz}$. Figures $1(\mathrm{a})$ and $1(\mathrm{~b})$ show the simulation and measurement setup of the FCH array. While a finite sized metal ground plane is used in the measurement, an infinitely large perfect electric conductor (PEC) ground is assumed in the simulation to save computational time. A voltage source is placed at the bottom of the left-most element and a field probe is moved in between parasitic elements to sample the near electric fields at the ground plane level for different frequencies. Numerical software FEKO [10] is used for simulation and a vector network analyzer (Agilent PNL N5230C) is applied for measurement.

Figure 2 compares the simulated and measured reflection coefficients $S_{11}$ of the source antenna. They exhibit similar trend and both are significantly different from the sharp resonance behavior of a single FCH antenna, as presented in [6]. This is due to the strong coupling between closely spaced helixes. The resonance band in the measurement shifts downward by approximately $20 \mathrm{MHz}$ comparing to the simulation data, which can be attributed to manufacturing errors.

Figures 3(a) and 3(b) show the normalized transmission data for both simulations and measurements on a $\mathrm{dB}$ scale. The antenna mismatches have been removed by normalizing $\left|S_{21}\right|^{2}$ to $1-\left|S_{11}\right|^{2}$ and $1-\left|S_{22}\right|^{2}$. The horizontal axis represents the frequency from $300 \mathrm{MHz}$ to $500 \mathrm{MHz}$ with an interval of $2.5 \mathrm{MHz}$, and the vertical axis represents the distance between transmitting and receiving antennas along the $y$-axis from $0.4 \mathrm{~m}$ to $1.6 \mathrm{~m}$ with a spacing of $0.08 \mathrm{~m}$. As shown in Figure 3(a), the receiving field strengths are much stronger 


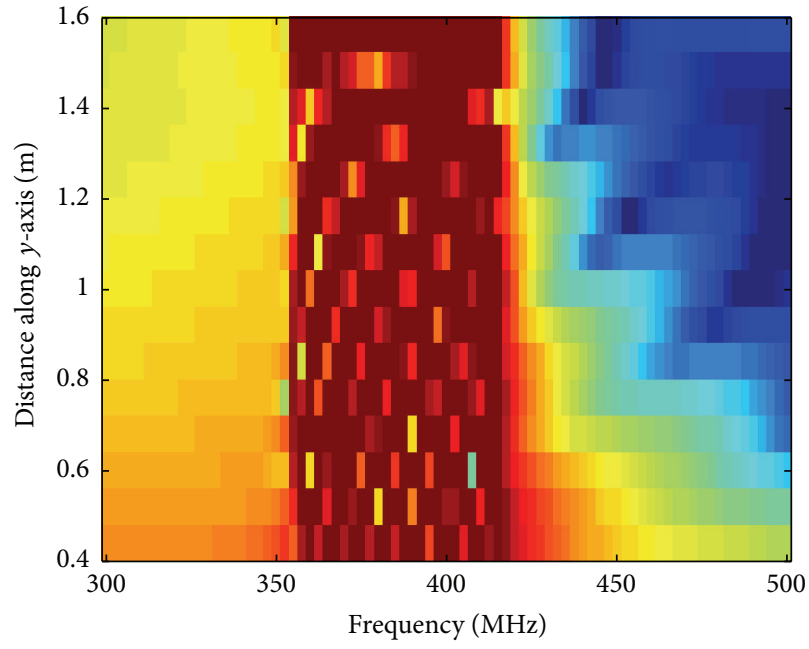

(a)

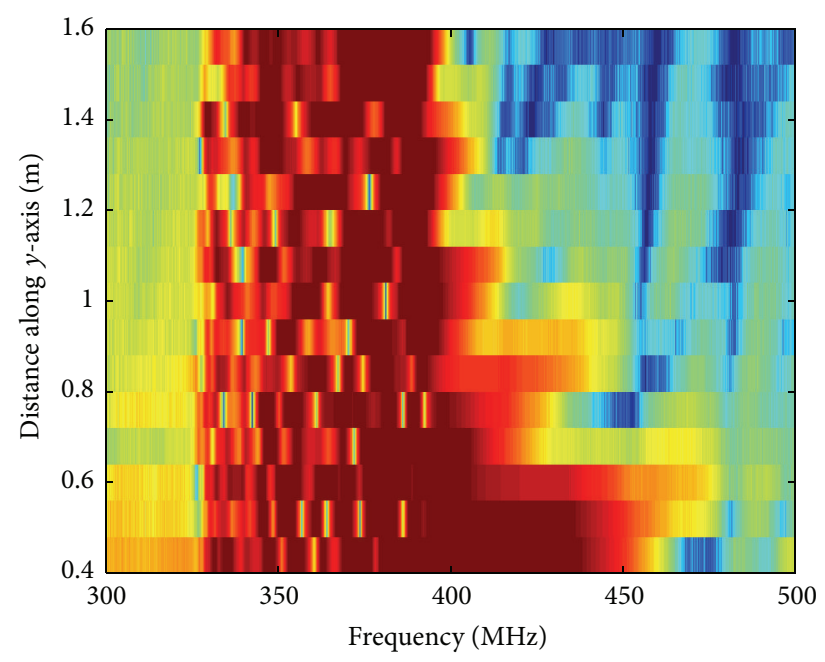

(b)

FIGURE 3: Normalized transmission coefficients: (a) simulation and (b) measurement.

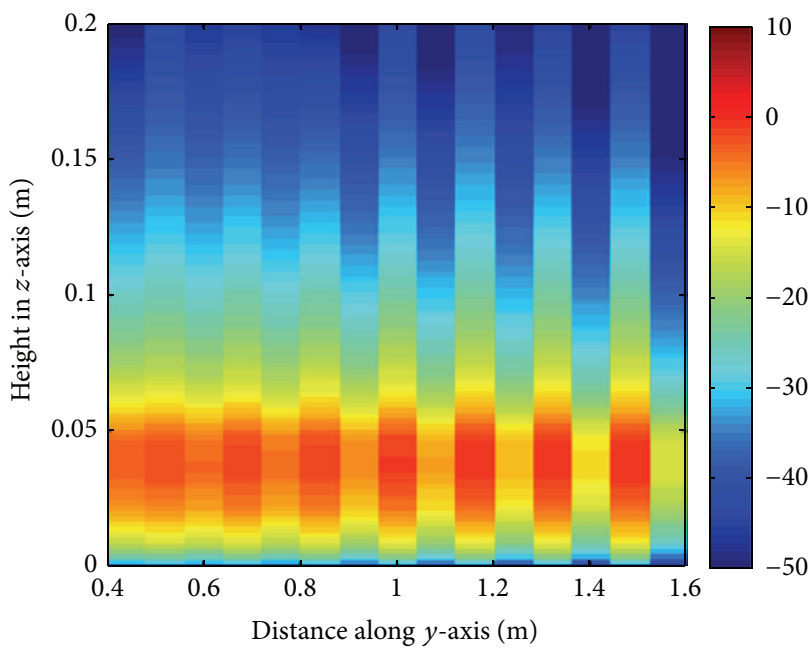

(a)

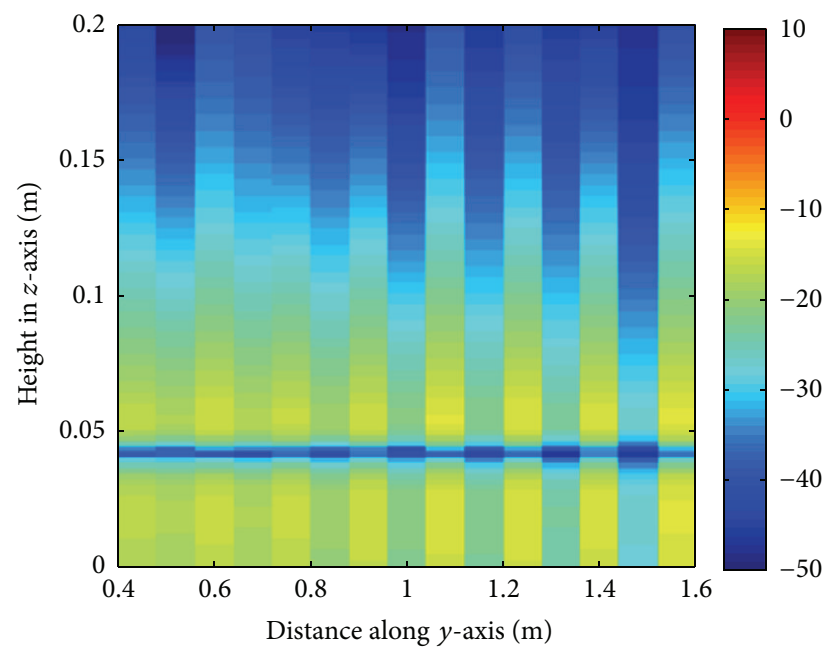

(b)

FIgURE 4: Simulated near field distribution in the $y-z$ plane at $390 \mathrm{MHz}$ : (a) $E_{y}$ and (b) $E_{z}$.

between $355 \mathrm{MHz}$ and $415 \mathrm{MHz}$, and within the pass band wave propagation exhibits negligible decay along the $y$ axis. Outside of this pass band, field strengths are much weaker and attenuate much faster. The measurement results in Figure 3(b) show good agreement with simulation results, except the pass band shifts downward by approximately $20 \mathrm{MHz}$, similar to the above observation for Figure 2.

To further reveal the wave propagation along the $\mathrm{FCH}$ array, we simulate the near field distributions in the $y-z$ plane at a sample frequency of $390 \mathrm{MHz}$. Figure 4 plots the normalized near electric field components $E_{y}$ and $E_{z}$ on a dB scale. The horizontal axis represents the distance along the $y$ axis from 0.4 to $1.6 \mathrm{~m}$ with a $0.08 \mathrm{~m}$ interval. The vertical axis represents height in the $z$ direction from 0 to $0.2 \mathrm{~m}$ with an interval of $0.002 \mathrm{~m}$. In Figure 4(a), a standing wave pattern can be clearly observed along the $y$ direction, implying the interference between $+y$ and $-y$ traveling waves due to the finite size of the array. Along the $z$ direction, the magnitude of $E_{y}$ peaks at the height of the helix and decays rapidly away from the interface between the $\mathrm{FCH}$ and the air. A similar interference pattern can be observed in Figure 4(b) for the $E_{z}$ component, except its strength is much weaker than $E_{y}$.

\section{Extraction of Dominant Wave Mechanism Using ESPRIT}

To better understand how wave propagates along the $1 \mathrm{D}$ FCH array, we extract the dominant wave mode and its associated propagation characteristics from the above transmission and near field data. First we model the transmission 


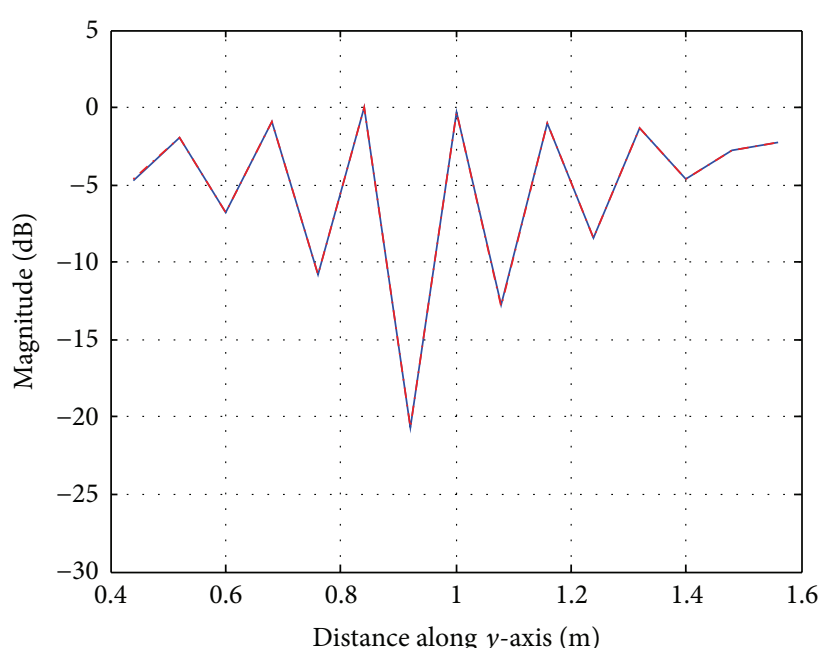

- Raw data by simulation

-..- Fitted curve by ESPRIT

(a)

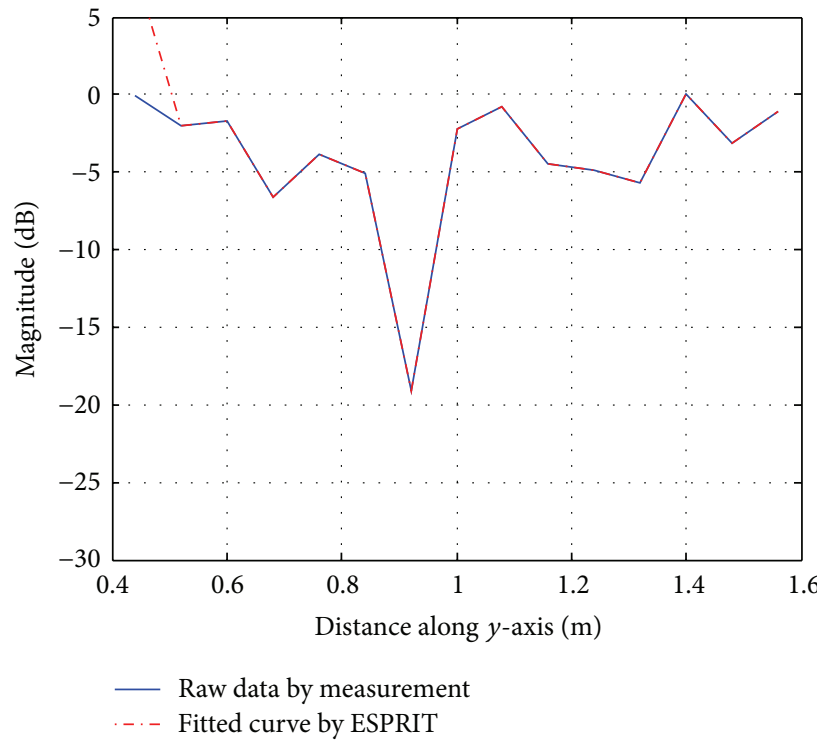

(c)

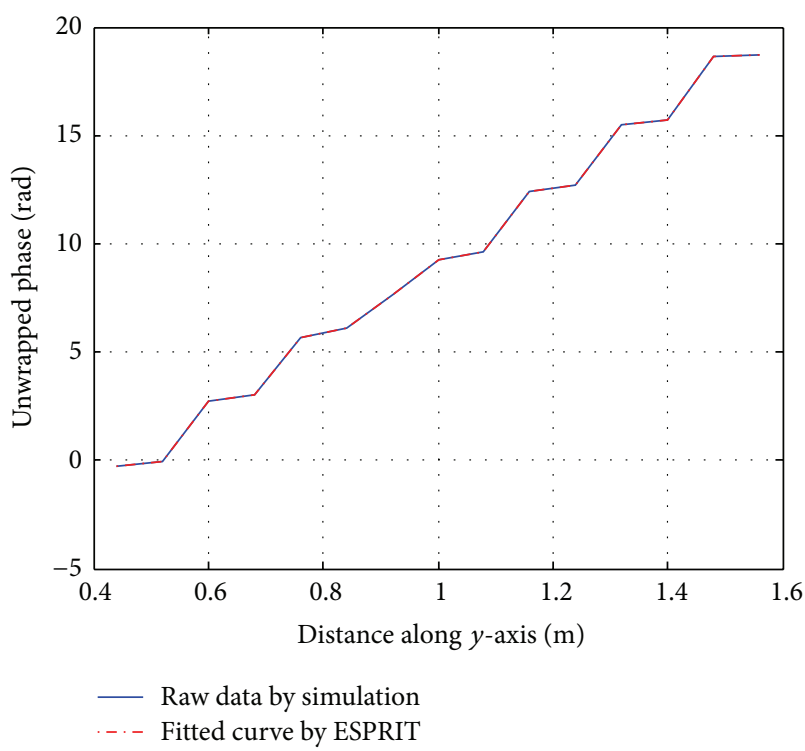

(b)

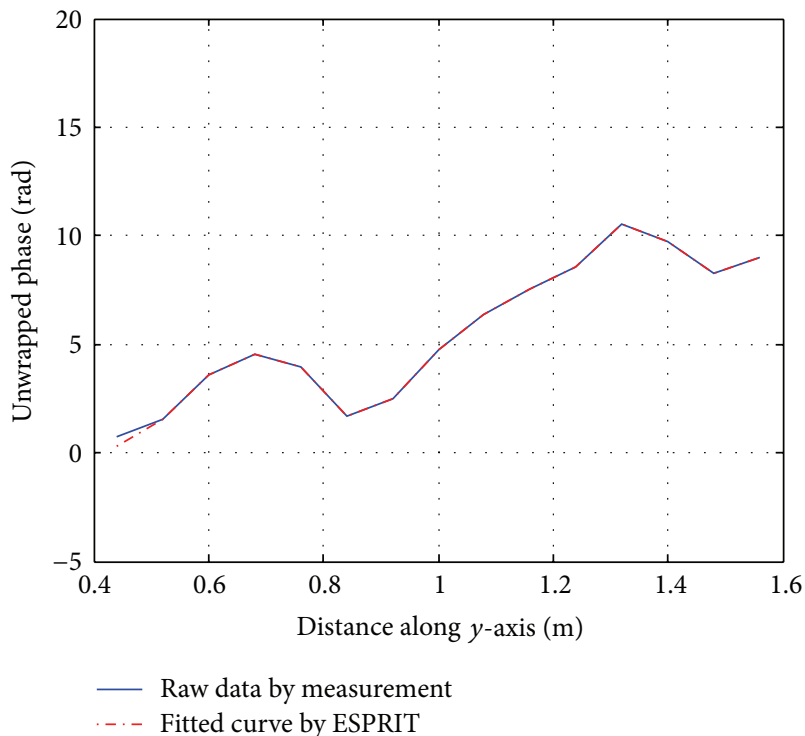

(d)

FIGURE 5: Comparison between original data with fitted results by ESPRIT: (a) simulated magnitude at $390 \mathrm{MHz}$; (b) simulated unwrapped phase at $390 \mathrm{MHz}$; (c) measured magnitude at $370 \mathrm{MHz}$; (d) measured unwrapped phase at $370 \mathrm{MHz}$.

data as a summation of different wave modes, each with a unique propagation constant $\beta_{m}$, attenuation constant $\alpha_{m}$, and magnitude $c_{m}$, which can then be extracted using ESPRIT algorithm. ESPRIT is a super resolution spectrum estimation algorithm which was originally developed for estimation of sinusoid signals in noise [11]. More recently, it has been successfully applied to both wave propagation and antenna radiation problems [12]. The extraction process has been explained in detail in [4] and will not be repeated here. Consider the following:

$$
S_{21} \cong \sum_{m=1}^{M} c_{m} e^{-j \beta_{m} y-\alpha_{m} y} .
$$

As an example, Figures 5(a) and 5(b) plot the ESPRIT fitted magnitude and phase curves at $390 \mathrm{MHz}$ by adding the first two dominant terms. The results show excellent agreement with the original simulated transmission data, and the propagation constants of the two dominant terms are found to be $-2.236 k_{0}$ and $2.236 k_{0}$ ( $k_{0}$ is the free space wave number), implying the superposition of incident and reflected components of a single slow wave mode. It is found that the dominant mode is a backward traveling surface wave for the following two reasons: first, the amplitude of the mode with a propagation constant $\beta=-2.236 k_{0}$ is stronger than other modes extracted from the ESPRIT; second, the unwrapped phase increases as distance $y$ increases, 


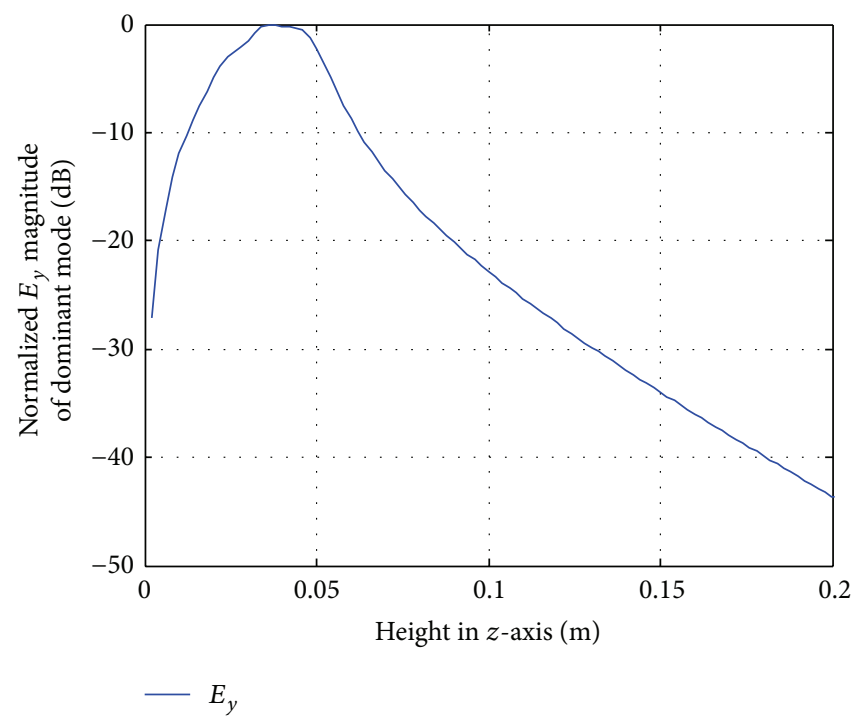

(a)

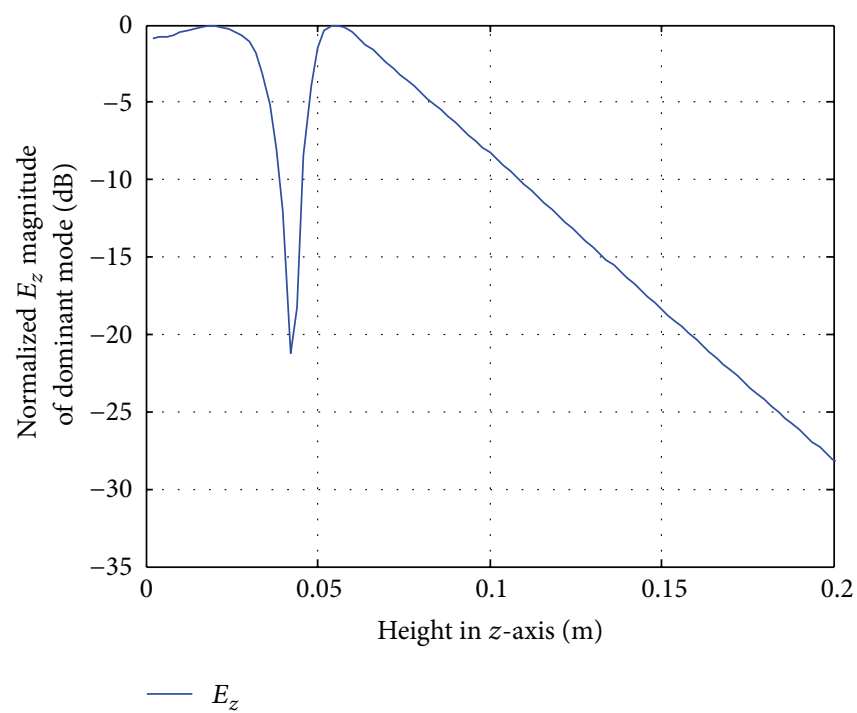

(b)

FIGURE 6: Normalized electric field mode distributions at $390 \mathrm{MHz}$ : (a) $E_{y}$ and (b) $E_{z}$.

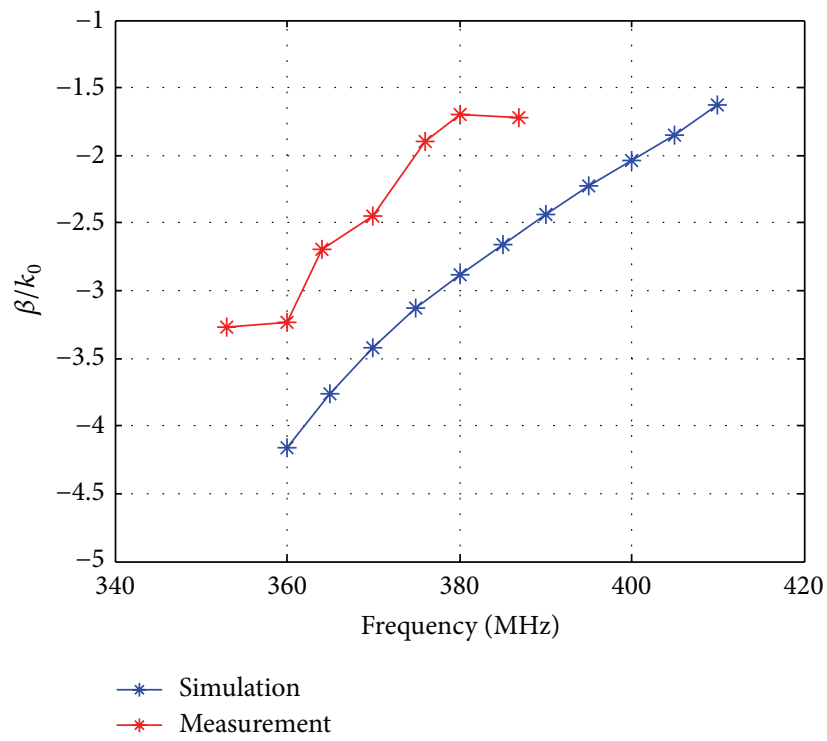

FIGURE 7: Extracted phase constants of simulation and measurement against frequency.

as shown in Figure 5(b), implying that the dominant mode is a backward traveling wave. The ESPRIT algorithm is also applied to the measured transmission data at $370 \mathrm{MHz}$ and the comparison between original and fitted data is shown in Figures 5(c) and 5(d). We intentionally shift the frequency downward by $20 \mathrm{MHz}$ to make a fair comparison between simulation and measurement, as stated in Section 2. Table 1 shows the propagation constants of the dominant backward surface wave, and the results are very similar between simulation and measurement.

Furthermore, we extract the mode distribution of the dominant surface wave from the simulated near electric field data in Figures 6(a) and 6(b). Figures 6(a) and 6(b) plot
TABLE 1: Propagation parameters of the dominant mode.

\begin{tabular}{lcc}
\hline Dominant mode & $\beta_{m} / k_{0}$ & $\alpha_{m} / k_{0}$ \\
\hline Simulation $(390 \mathrm{MHz})$ & -2.236 & 0.0089 \\
\hline Measurement $(370 \mathrm{MHz})$ & -2.4485 & 0.1584 \\
\hline
\end{tabular}

the extracted $E_{y}$ and $E_{z}$ mode distributions versus height $z$ at $390 \mathrm{MHz}$. It can be seen that the magnitude of $E_{y}$ peaks around the height of the $\mathrm{FCH}$ element $(0.047 \mathrm{~m})$ and decays exponentially away from the interface, a typical characteristic of surface wave propagation. The $E_{z}$ distribution is more complicated below the interface between the $\mathrm{FCH}$ and the air 


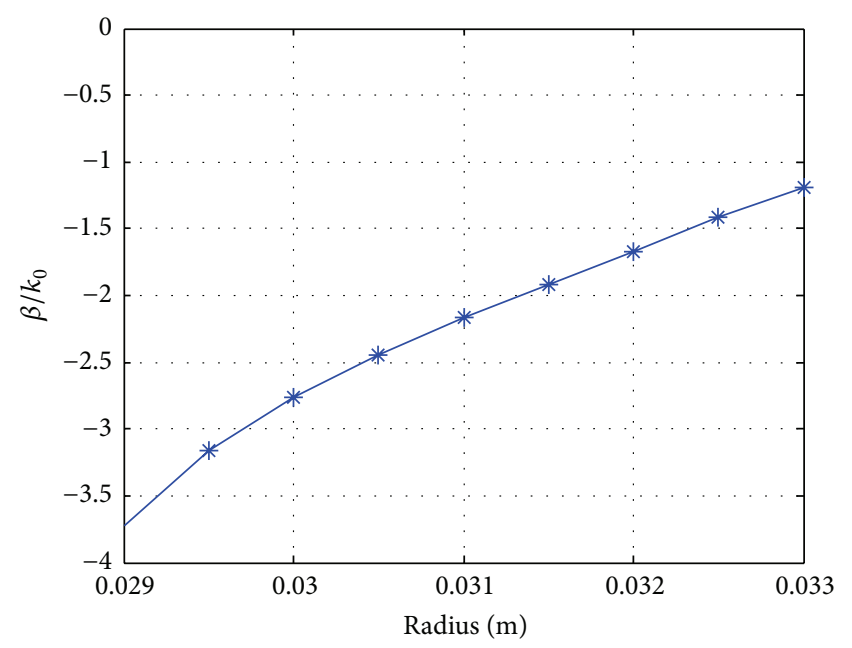

(a)

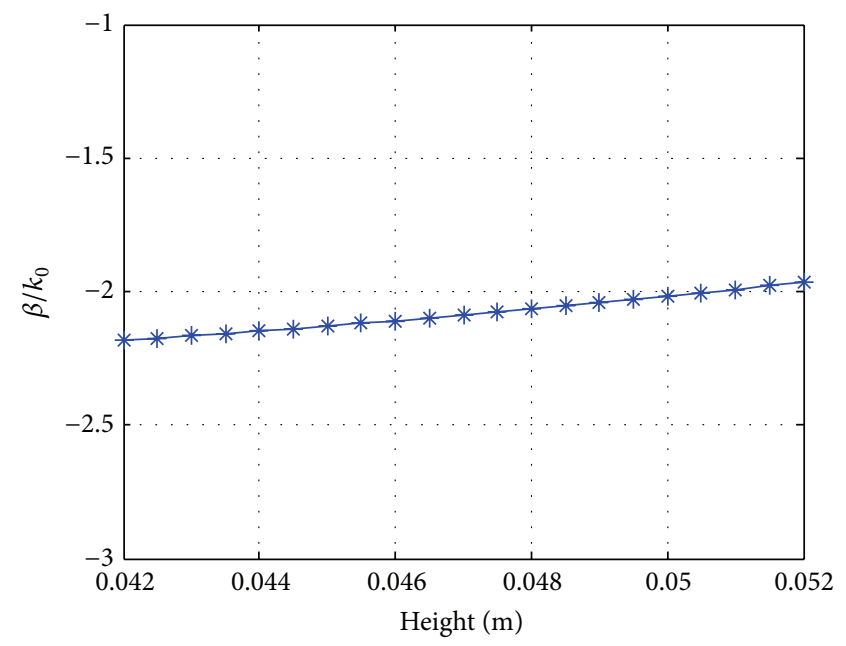

(b)

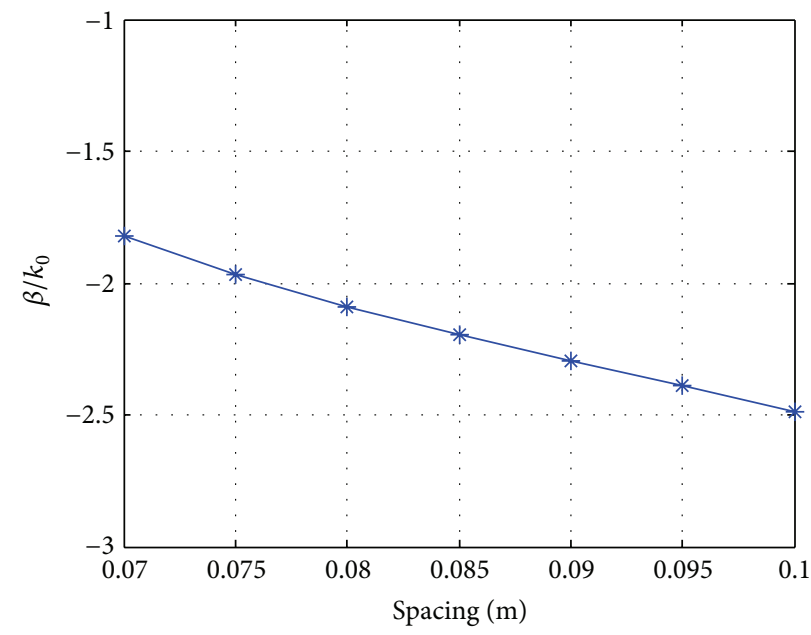

(c)

FIGURE 8: Parametric study on array geometry parameters: (a) radius; (b) height; (c) spacing.
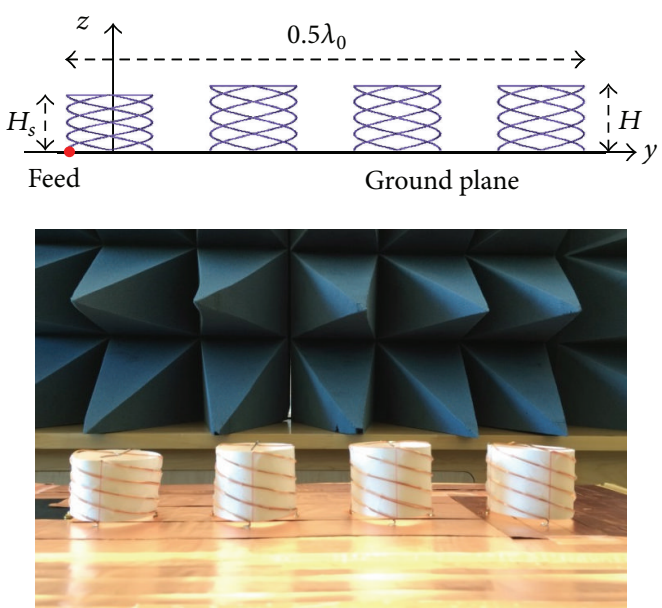

FIGURE 9: 1D compact, closely spaced backfire antenna array. 


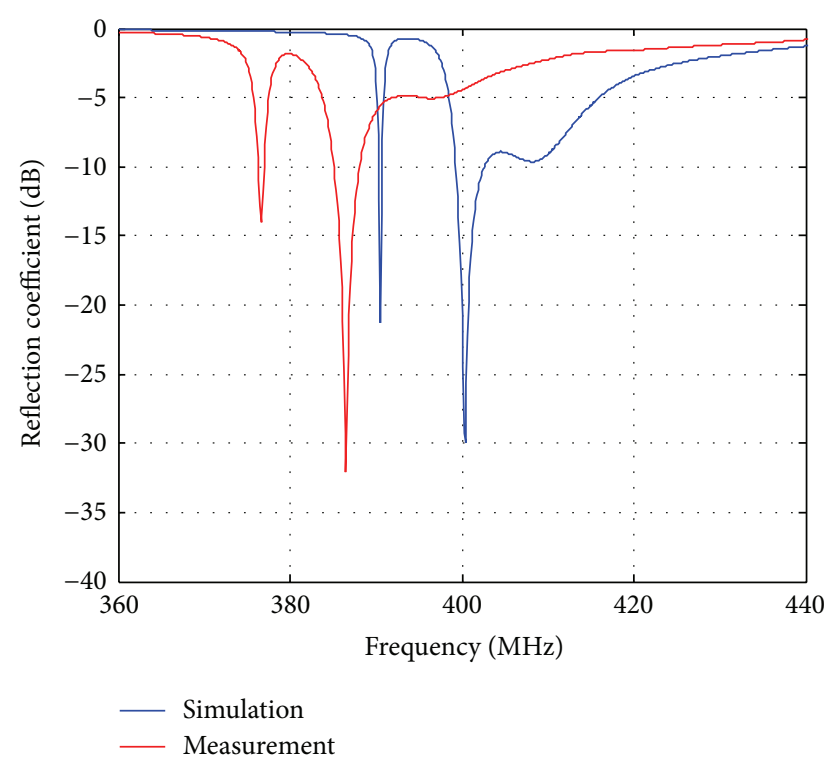

FIGURE 10: Simulated and measured antenna reflection coefficients.

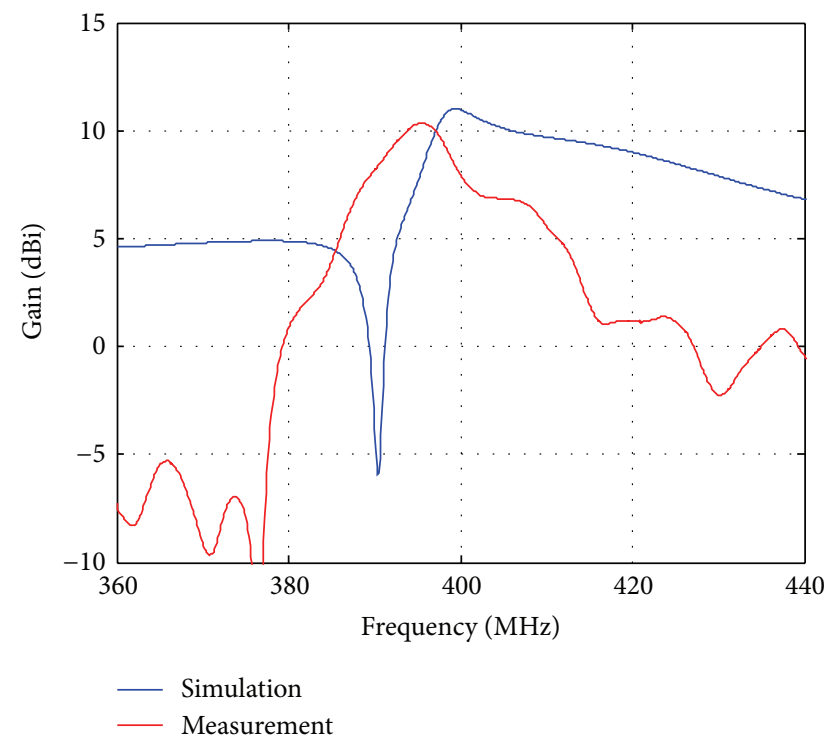

FIGURE 11: Simulated and measured antenna gain.

but is similar to $E_{y}$ above the FCH height. We also map out the frequency dispersion behavior of the backward traveling surface wave and show the results in Figure 7. It is seen that, except for the $20 \mathrm{MHz}$ frequency shift along the horizontal axis, the values of $\beta$ increase as frequency increases for both simulations and measurements.

Finally, a parametric study is conducted to correlate the propagation constant with array geometrical parameters. The first parameter we examined is the radius $R$. Both the height and the spacing between elements are fixed. Figure 8(a) shows that $\beta$ increases as $R$ increases. The second varying parameter is the height $H$ of the helix, as shown in Figure $8(\mathrm{~b})$. The phase constant varies slightly since the total length of the helix does not change much. Finally, in
Figure 8(c) we explored the effect of spacing and it was found that by increasing the interval of the adjacent element from $0.07 \mathrm{~m}$ to $0.1 \mathrm{~m}$ the propagation constant drops from $-1.83 k_{0}$ to $-2.49 k_{0}$ due to the variation of coupling between array elements. It is concluded that by carefully selecting a combination of radius $R$, height $H$, and spacing $S$ we can achieve a desired propagation constant $\beta$ for a fixed length FCH array. This observation is rather important for the surface wave antenna design, as will be explained in the next section.

\section{Backfire Surface Wave Antenna Design}

In this section, a compact $1 \mathrm{D}$, closely spaced parasitic antenna array centered at $400 \mathrm{MHz}$ is designed based on the above identified dominant surface wave mechanism. The total length of the array is assumed to be $0.5 \lambda_{0}$. Given this fixed array size, the propagation constant of the surface wave has to be carefully determined to maximize the directivity of the array, which is similar to the $1 \mathrm{D}$ metal wire antenna array design [4]. The optimum phase constant $\beta_{\text {opt }}$ for this halfwavelength array is found to be $-1.98 k_{0}$. Then the radius $R$, height $H$, and spacing $S$ are selected to be $0.03115 \mathrm{~m}, 0.049 \mathrm{~m}$, and $0.104 \mathrm{~m}$ to achieve this $\beta_{\text {opt }}$. Other combinations of array geometrical parameters can also result in this optimum propagation constant, as discussed in the previous parametric study. Figure 9 plots the final array design setup, which consists of four FCH elements. The height of the source antenna is tuned to be $0.042 \mathrm{~m}$ to match the input impedance to $50 \mathrm{ohms}$ at the center frequency.

Figure 10 shows the antenna reflection coefficients versus frequency. The center resonance frequencies are found to be $400 \mathrm{MHz}$ and $386 \mathrm{MHz}$ for simulation and measurement. The $-10 \mathrm{~dB}$ bandwidthes are both $3 \mathrm{MHz}$. Figure 11 compares the simulated and measured gain in the backward end-fire direction $\left(\theta=90^{\circ}, \phi=270^{\circ}\right)$. The maximum gain values are $10.99 \mathrm{dBi}$ for simulation and $10.33 \mathrm{dBi}$ for measurement. Finally, Figure 12 plots the simulated antenna array radiation patterns in both the azimuth plane (i.e., $x y$ plane) and the elevation plane (i.e., $y z$ plane) at the center frequency, which clearly shows the backward radiation pattern. The simulated front-to-back ratio is found to be $9.4 \mathrm{~dB}$, while in measurement it is $12.8 \mathrm{~dB}$.

To provide more physical insights into our $\mathrm{FCH}$ array operation, we compare its interelement phase delay with the well-known Hansen-Woodyard end-fire array condition [13]. Given the extracted propagation constant $\left(-1.98 k_{0}\right)$ and spacing $\left(0.138 \lambda_{0}\right)$, the phase delay $\theta_{0}$ between adjacent FCH elements is found to be

$$
\theta_{0}=\beta_{\mathrm{opt}} d=-1.98 k_{0} \times 0.138 \lambda_{0}=-1.72 \mathrm{rad} .
$$

According to the Hansen-Woodyard end-fire array condition, the theoretical optimum phase delay $\theta_{\mathrm{HW}}$ between adjacent array elements should be close to

$$
\begin{aligned}
\theta_{\mathrm{HW}} & =-\left(k_{0} d+\frac{\pi}{N}\right)=-\left(\frac{2 \pi f d}{c}+\frac{\pi}{N}\right) \\
& =-1.6525 \mathrm{rad},
\end{aligned}
$$


TABLE 2: Comparison between our antenna and other small antenna arrays in the literatures.

\begin{tabular}{|c|c|c|c|c|c|c|}
\hline Reference & Number of elements & Spacing & Height & Gain & $\mathrm{F} / \mathrm{B}$ & BW \\
\hline Our antenna & 4 & $0.138 \lambda_{0}$ & $0.06 \lambda_{0}$ & $10.3 \mathrm{dBi}$ & $12.8 \mathrm{~dB}$ & $0.75 \%$ \\
\hline$[14]$ & 3 & $0.11 \lambda_{0}$ & $0.275 \lambda_{0}$ & $7.14 \mathrm{dBi}$ & $8.6 \mathrm{~dB}$ & $2.28 \%$ \\
\hline$[15]$ & 3 & $0.06 \lambda_{0}$ & $0.092 \lambda_{0}$ & $8.5 \mathrm{dBi}$ & $7 \mathrm{~dB}$ & $2.7 \%$ \\
\hline$[16]$ & 3 & $0.09 \lambda_{0}$ & $0.1 \lambda_{0}$ & $8.43 \mathrm{dBi}$ & $6.63 \mathrm{~dB}$ & $1.2 \%$ \\
\hline [17] & 3 & $0.053 \lambda_{0}$ & $0.21 \lambda_{0}$ & $9.9 \mathrm{dBi}$ & $\mathrm{n} / \mathrm{a}$ & $12.44 \%$ \\
\hline
\end{tabular}

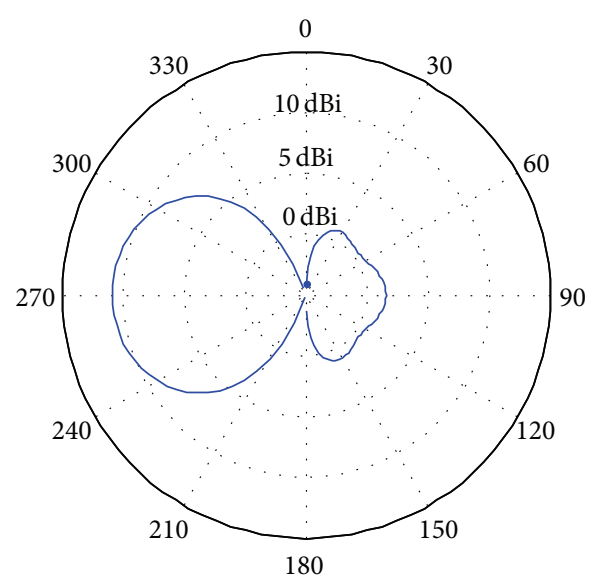

(a)

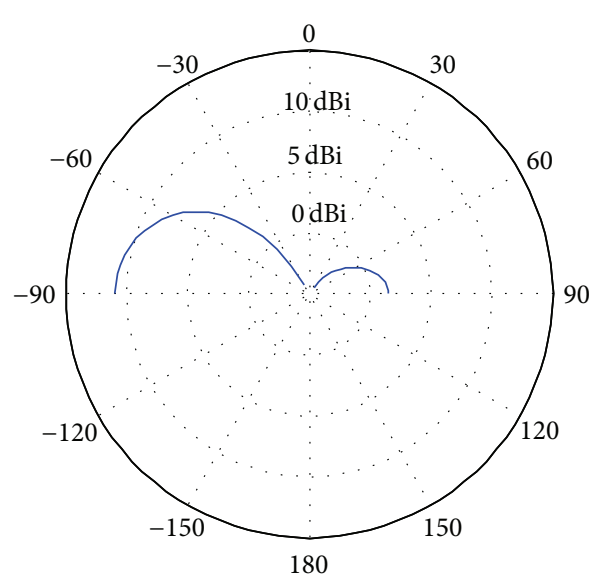

(b)

FIGURE 12: Simulated radiation patterns at $400 \mathrm{MHz}$ : (a) azimuth plane and (b) elevation plane.

where $c$ is speed of light and $N$ is number of elements. It can be seen thatthe phase delay of our FCH array $\theta_{0}$ is close to that of Hansen-Woodyard end-fire condition $\theta_{\mathrm{HW}}$, resulting in its maximum radiation in the $-y$ direction.

Finally, we compare our FCH antenna array performance with other small antenna arrays in the literatures [14-17]. The comparison is shown in Table 2 . It can be clearly seen that our FCH array exhibits the lowest height, highest gain, and best front-to-back (F/B) ratio among all antennas. The tradeoffs we made include one more element and slightly larger spacing. The $-10 \mathrm{~dB}$ input bandwidth of our antenna is the smallest, which can be attributed to the lowest height of the FCH element.

\section{Conclusion}

In this paper, surface wave propagation and radiation along a 1D FCH array is investigated from both simulation and measurement perspectives. It is found that a backward traveling surface wave can be supported by this structure. The propagation characteristics of this dominant wave mechanism are extracted and discussed. Furthermore, a backfire surface wave antenna is designed and implemented based on the identified wave mechanism. The study presented in this paper opens a new door for low-profile parasitic surface wave antenna array design. For future work, we will expand the study to array structures with other electrically small elements such as the folded spherical helix or the meander line. We will also look into the possibility of applying the $\mathrm{FCH}$ antenna array for wireless power transfer.

\section{Conflict of Interests}

The authors declare that there is no conflict of interests regarding the publication of this paper.

\section{References}

[1] G. Thiele, "Analysis of yagi-uda-type antennas," IEEE Transactions on Antennas and Propagation, vol. 17, no. 1, pp. 24-31, 1969.

[2] H. W. Ehrenspéck and H. Poehler, "A new method for obtaining maximum gain from yagi antennas," IRE Transactions on Antennas and Propagation, vol. 7, no. 4, pp. 379-386, 1959.

[3] S. A. Maier, M. L. Brongersma, and H. A. Atwater, "Electromagnetic energy transport along arrays of closely spaced metal rods as an analogue to plasmonic devices," Applied Physics Letters, vol. 78, no. 1, pp. 16-18, 2001.

[4] D. Xue and Y. Li, "Design of a closely-spaced, multiple-element parasitic antenna array," Microwave and Optical Technology Letters, vol. 56, no. 10, pp. 2374-2377, 2014.

[5] S. R. Best, "The radiation properties of electrically small folded spherical helix antennas," IEEE Transactions on Antennas and Propagation, vol. 52, no. 4, pp. 953-960, 2004.

[6] S. R. Best, "A comparison of the cylindrical folded helix Q to the Gustafsson limit," in Proceedings of the 3rd European Conference on Antennas and Propagation (EuCAP '09), pp. 2554-2557, Berlin, Germany, March 2009.

[7] M. Škiljo and Z. Blažević, "Spherical helices for resonant wireless power transfer," International Journal of Antennas and Propagation, vol. 2013, Article ID 426574, 12 pages, 2013.

[8] J. Lee and S. Nam, "Fundamental aspects of near-field coupling small antennas for wireless power transfer," IEEE Transactions 
on Antennas and Propagation, vol. 58, no. 11, pp. 3442-3449, 2010.

[9] B. Xu, R. Jean, and Y. Li, "Investigation of surface wave propagation along a folded cylindrical helix array," in Proceedings of the IEEE Texas Symposium on Wireless and Microwave Circuits and Systems (WMCS '15), Waco, Tex, USA, April 2015.

[10] https://www.feko.info/.

[11] A. Paulraj, R. Roy, and T. Kailath, "Estimation of signal parameters via rotational invariance techniques-ESPRIT," in Proceedings of the 19th Asilomar Conference on Circuits, Systems and Computers, pp. 83-89, November 1985.

[12] Y. Li and H. Ling, "Extraction of wave propagation mechanisms in a cut-wire array using the ESPRIT algorithm," IEEE Antennas and Wireless Propagation Letters, vol. 8, pp. 744-747, 2009.

[13] W. L. Stutzman and G. A. Thiele, Antenna Theory and Design, John Wiley \& Sons, 2012.

[14] Z. Bayraktar, P. L. Werner, and D. H. Werner, "The design of miniature three-element stochastic Yagi-Uda arrays using particle swarm optimization," IEEE Antennas and Wireless Propagation Letters, vol. 5, no. 1, pp. 22-26, 2006.

[15] S. Lim and H. Ling, "Design of electrically small, pattern reconfigurable Yagi antenna," Electronics Letters, vol. 43, no. 24, pp. 1326-1327, 2007.

[16] J. Yu, Y. Le, and S. Lim, "Design of a dual-band, electrically small, parasitic array antenna," IEEE Antennas and Wireless Propagation Letters, vol. 13, pp. 1453-1456, 2014.

[17] D. Arceo and C. A. Balanis, "A compact Yagi-Uda antenna with enhanced bandwidth," IEEE Antennas and Wireless Propagation Letters, vol. 10, pp. 442-445, 2011. 

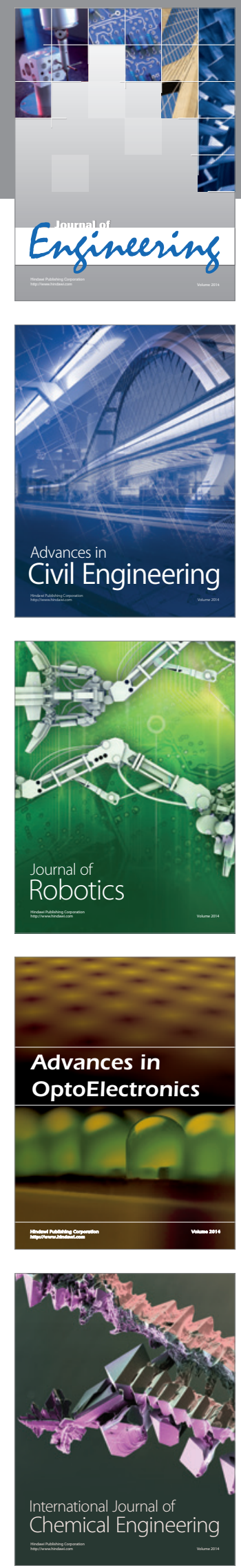

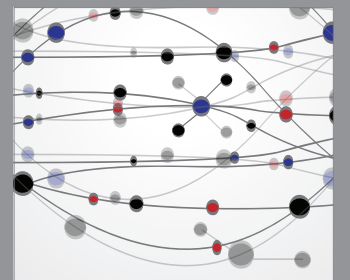

The Scientific World Journal
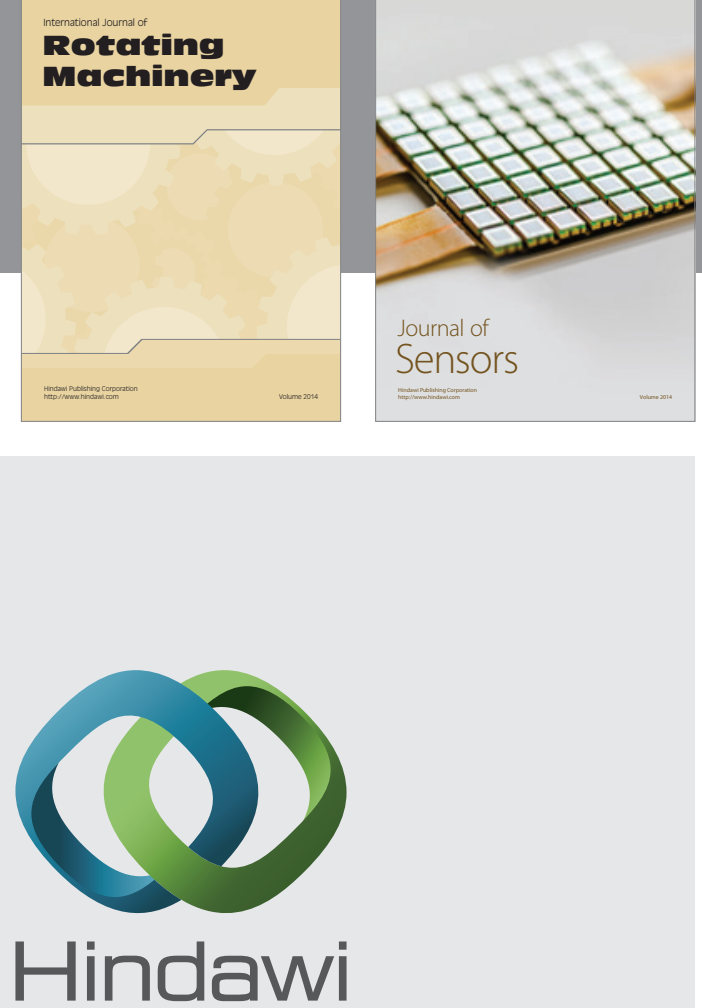

Submit your manuscripts at http://www.hindawi.com
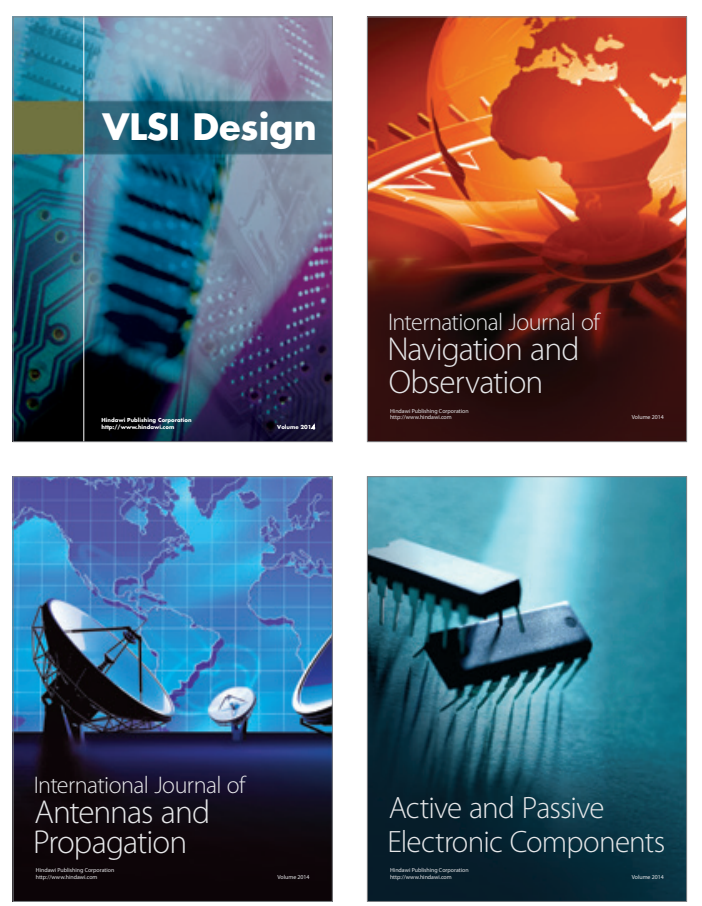
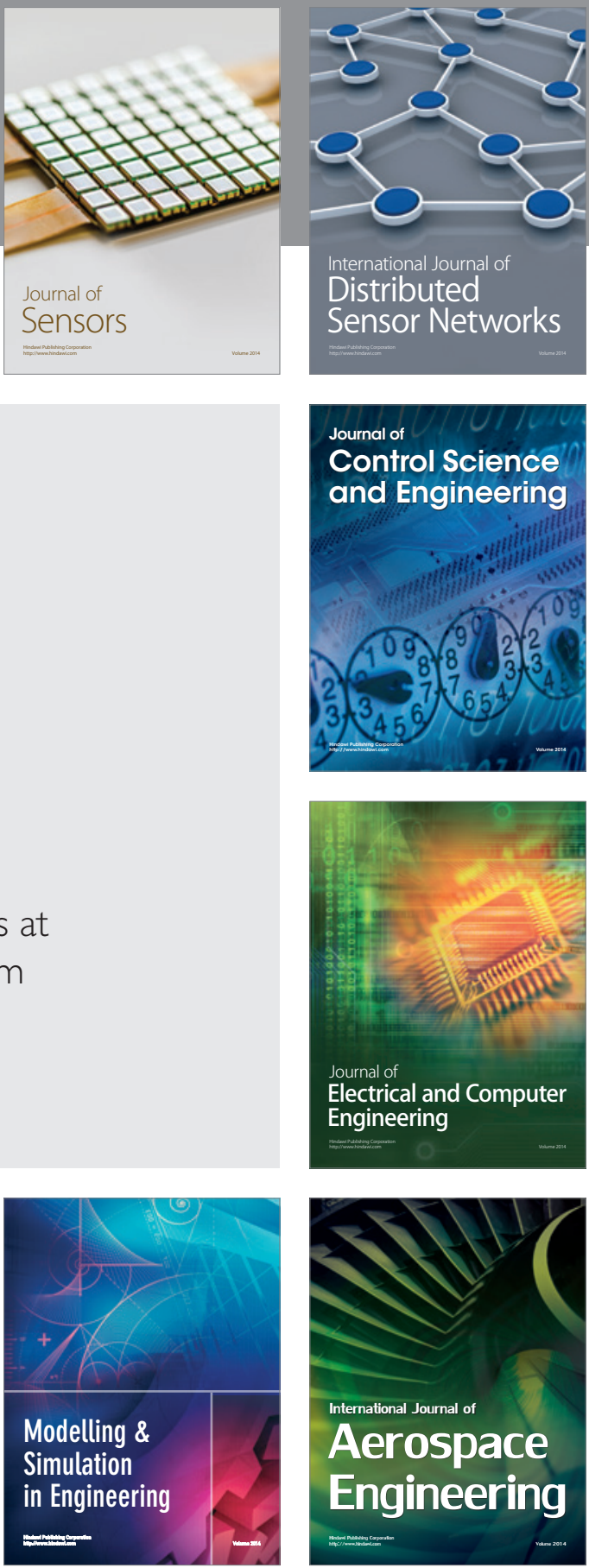

Journal of

Control Science

and Engineering
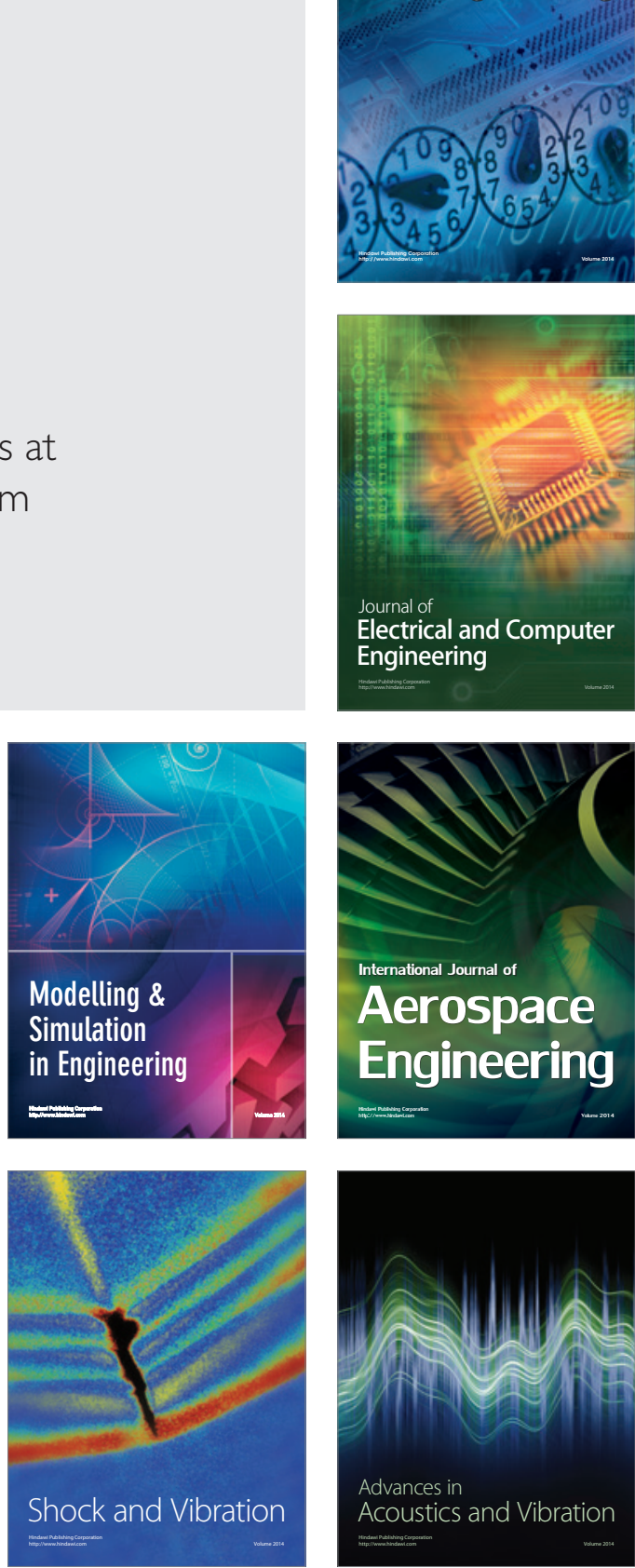